\title{
LATERAL IN-PLANE DISPLACEMENT MICROACTUATORS WITH COMBINED THERMAL AND ELECTROSTATIC DRIVE
}

\author{
Xi-Qing Sun, Xiaoyi Gu, and W. N. Carr \\ Microelectronics Research Center, New Jersey Institute of Technology \\ Room 200-MIC, 323 King Blvd., Newark, NJ07102, USA \\ sun@hertz.njit.edu, carr@admin.njit.edu
}

\begin{abstract}
Electrostatic actuation using a bimorph structure has been combined with thermal actuation in cantilever devices. The combined electrostatic and electrothermal excitation increases the efficiency of the actuation mechanism and permit a 12 volt voltage supply and $40 \mathrm{~mW}$ power. In this work we have optimized the design to create a horizontal movement of $\Delta x=60$ $\mu \mathrm{m}$ for cantilever of overall initial length $\mathrm{L}=1000 \mu \mathrm{m}$. The actuation characteristics of cantilevers with only electrostatic actuation, only thermal actuation, and combined actuation are separately presented for comparison. These actuators are designed for direct interface to CMOS driver circuits.
\end{abstract}

\section{INTRODUCTION}

Thermal and electrostatic actuators have mostly been described for out-of-plane displacements. These devices have included the micro tweezers [1,2], micro polymeric-actuators [3], and micromanipulators [4]. The work of Keller and Howe describes one of the few thermally-actuated devices with inplane actuation [5]. Our present work is with reversed bimorph structures, i.e., the top layer with a smaller thermal expansion coefficient and the bottom layer with a larger value. Following the sacrificial layer release, the cantilever bows upwards due to the built-in stress. Our device exhibits an actuation similar to an "inchworm" to achieve a lateral in-plane displacement of its tip along the plane of the underlying insulation/substrate surface.

When electrostatic actuation is used alone, the device voltage supply is generally 50 volts and higher $[1,3,6]$. When thermal heating alone is used as the actuating mechanism, the power required is in the range of hundreds of milliwatts $[3,4]$. These voltage and power requirements limit the interface compatibility for VLSI device circuits especially when low power is a requirement. By combining the thermal and electrostatic drive we have reduced the voltage requirement to 12 volts and the power requirement to $40 \mathrm{~mW}$. These levels are compatible with CMOS single chip drivers.

\section{DESIGN AND FABRICATION}

The inchworm actuator is schematically shown in Fig.1. As a cantilever structure with a fixed end, initially the released cantilever bows upwards over hundreds microns of out-of-plane displacement at its center and several tens of in-plane displacement at its tip as illustrated in Fig.2 (a). As an excitation either thermal $V_{a}$ or electrostatic $V_{b}$ is imposed on the actuator, the up-bowed cantilever stretches out along the insulation surface in Fig.2 (b), and moves up to the fully closed state in Fig. 2 (c) as the excitation exceeds a threshold value.
Throughout the whole process, the tip part always keeps contact with the substrate, and thus the inchworm actuator can achieve lateral in-plane displacement. It is because of this point, a friction between the moving ip and the insulation surface is importantly involved in this approach.

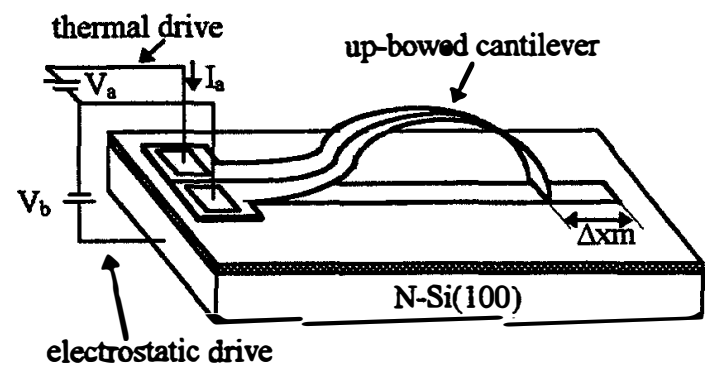

Fig.1. A schematic view of up-bowed actuator.

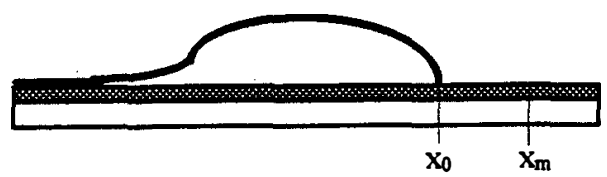

(a) initial up-bowed position $(\mathrm{Va}=0, \mathrm{Vb}=0)$

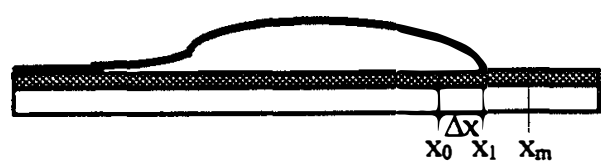

(b) intermediate partially actuated position

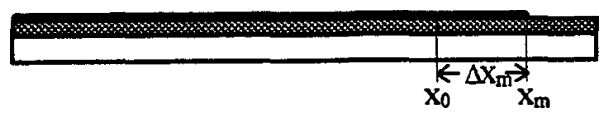

(c) fully actuated closed position

Fig.2. Actuation sequence illustration.

Fig. 3 shows the schematic views of the inchworm actuator, which is a polysilicon layer on a $\mathrm{TaSi}_{2} / \mathrm{Si}_{3} \mathrm{~N}_{4}$ composite layer to form the bimorph structure. The thermal coefficients of polysilicon, $\mathrm{TaSi}_{2}$ and $\mathrm{Si}_{3} \mathrm{~N}_{4}$ are $2.33 \times 10^{-6} / \mathrm{K}, 8.8 \times 10^{-6} / \mathrm{K}$ and $2.8 \times 10^{-6} / \mathrm{K}$ respectively. The high-temperature-balanced bimorph will spring a large curvature at low temperature as soon 
as it is released [7]. The polysilicon and $\mathrm{TaSi}_{2}$ layers serve as the heater for thermal actuation and upper electrode for electrostatic actuation. The $\mathrm{Si}_{3} \mathrm{~N}_{4}$ layer offers protection for the upper $\mathrm{TaSi}_{2}$ layer from $\mathrm{HF}$ solution. A $\mathrm{Si}_{3} \mathrm{~N}_{4} / \mathrm{SiO}_{2}$ composite layer is used as an insulation layer between the actuator and the substrate, and also provides the stationery support film for the actuator tip motion.

(a)

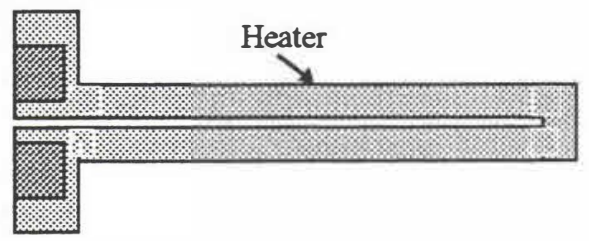

(b)

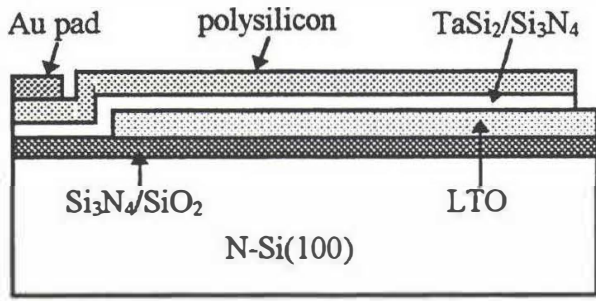

Fig.3. Schematic view: (a) top; (b) side.

The fabrication process started by oxidizing $100 \mathrm{~nm}$ thick $\mathrm{SiO}_{2}$ on a n-type (100) silicon wafer. This is followed by an LPCVD $300 \mathrm{~nm} \mathrm{Si} \mathrm{Si}_{4}$ layer as an insulation and HF-resistant layer. Next, a $2 \mu \mathrm{m}$ thick LTO was deposited and pattemed as

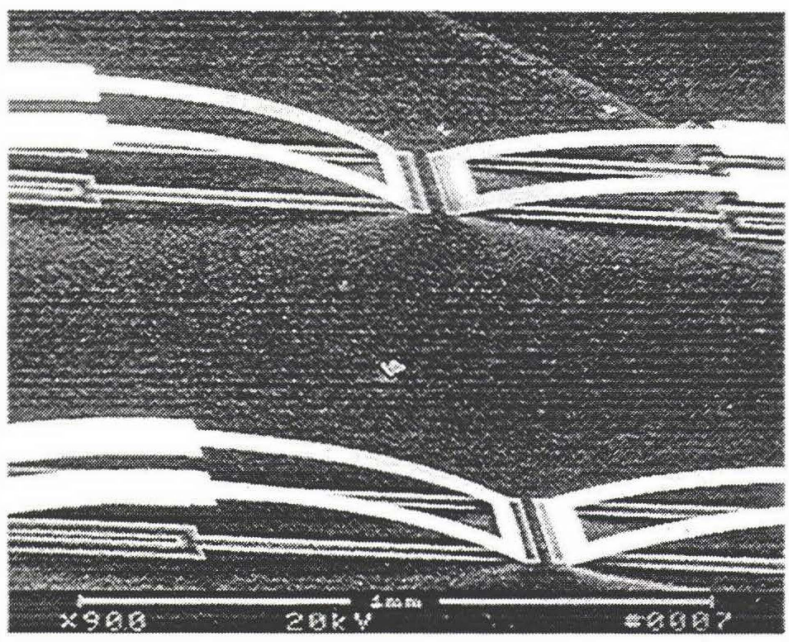

Fig.4. SEM photograph of an up-bowed microactuator.

sacrificial layer. After the $200 \mathrm{~nm} \mathrm{Si} \mathrm{N}_{4}$ deposition, we followed with a $100 \mathrm{~nm} \mathrm{TaSi}{ }_{2}$ sputtering, a $800 \mathrm{~nm}$ polysilicon deposition, a subsequent phosphorus diffusion at $950{ }^{\circ} \mathrm{C}$, photolithography and $\mathrm{RIE}$ in that order. The $\mathrm{TaSi}_{2}$ material was selected not only for raising the thernal mismatch, but also for lowering the heater resistance to reduce the parasitic electrostatic influence during thermal actuation. The metal contact pads are a $500 \mathrm{~nm}$ gold layer. The final release step was done by dipping the wafers into $49 \% \mathrm{HF}$ solution for over 15 min. The released cantilever bows up and the movable tip

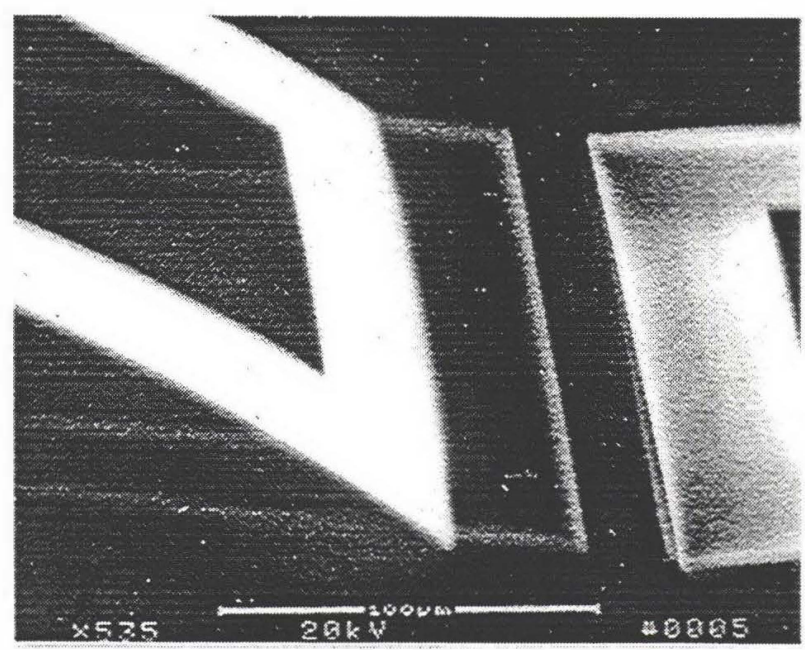

Fig.5. SEM photograph of the tip with an initial lateral in-plane displacement of 35 rom.

retracts backwards on the insulation surface as is shown in the SEM views of Fig.4 and Fig.5. Cantilevers with $1000 \mu \mathrm{m}$ length and $35 \mu \mathrm{m}$ width can shrink back 30 to $60 \mu \mathrm{m}$ lateral inplane displacement following release. Correspondingly, the vertical out-of-plane displacement of the center part is hundreds microns. So, the up-bowed actuator can generate both the lateral in-plane and the vertical out-of-plane displacements at the same time. In our current design, the inchworm actuator fabrication possesses high yield more than $80 \%$ without special release processing.

\section{TEST AND RESULTS}

\section{Electrostatic Actuation}

A CMOS high-voltage supply [8] was adopted to supply a voltage ranging from $0 \mathrm{~V}$ to $60 \mathrm{~V}$ for electrostatic drive. As the applied voltage increases, the up-bowed cantilever is attracted downward under the electrostatic attraction induced from the substrate. Consequently, the tip is pushed laterally along the substrate surface as it overcomes friction with the underlying insulation layer. A cantilever with $1000 \mu \mathrm{m}$ in length and $30 \mu \mathrm{m}$ initial lateral displacement was chosen in this test. The measured relationship between the lateral tip position and the applied voltage is shown in Fig. 6. Very similar to the parallel electrode attraction [9], the tip lateral displacement increases steadily as an increase in applied voltage initially. Once the bias exceeds a threshold $\mathrm{V}_{\mathrm{t}}$ at $53 \mathrm{~V}$, the cantilever suddenly closes and fully flattens to the insulation surface to achieve the full 
lateral displacement. As the bias sweeps down, a hysteresis is observed as shown in Fig. 6 which is contributed mainly by the nature of the parallel electrodes attraction [9], and partly by the friction between the tip and the insulation surface.

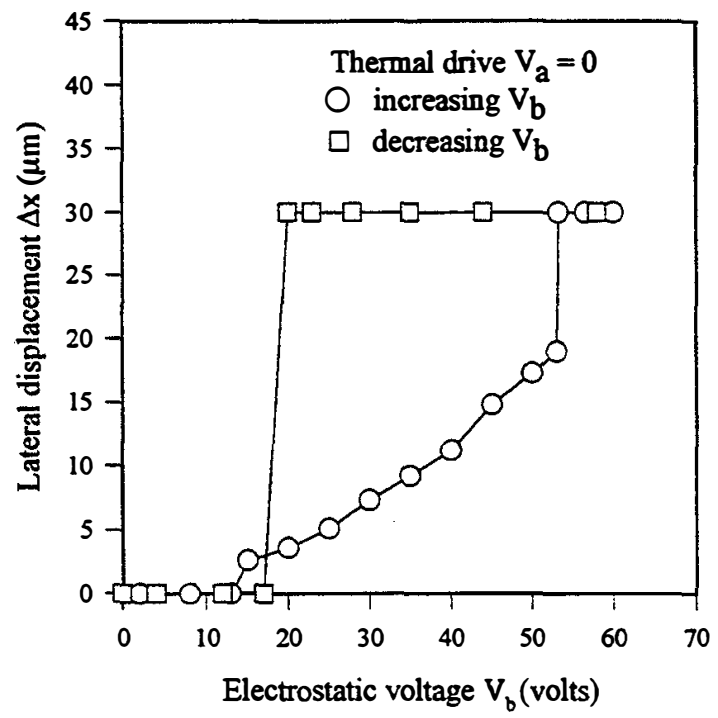

Fig.6. Measured lateral displacement as a function of applied voltage for electrostatic actuation only.

In some aspects, the electrostatically actuated inchworm is similar in principle to the microscratch reported previously[10]. But the up-bowed cantilever structure reported in this design can achieve much larger lateral in-plane displacement.

\section{Thermal Actuation}

Quite different from the electrostatic drive, thermal actuation is based on the thermal expansion mismatch of the heated bimorph. The thermal bimorph also provides large force and displacement. A current source $\mathrm{I}_{3}$ was used for thermal actuation. Fig. 7 shows the relationship of tip displacement versus input power. As can been seen, the up-bowed cantilever reaches a displacement of $26 \mu \mathrm{m}$ at the input power of $86 \mathrm{~mW}$ and above at approximate $650^{\circ} \mathrm{C}$.

In Fig. 7 there is a slight hysteresis between the power increasing and the power decreasing processes, probably due to the tip friction against the substrate.

\section{Combined Drive}

As described above, separate thermal and electrostatic drives require either high electrostatic voltage up to $53 \mathrm{~V}$ or high thermal power up to $86 \mathrm{~mW}$ to achieve the maximum motion. In this example, a constant electrostatic bias of $12 \mathrm{~V}$ was introduced to assist the thermal actuation. Fig. 8 shows the measured results of the combined drive. As can been seen, the input thermal power and the applied electrostatic voltage at which the actuator achieves the full range motion are $40 \mathrm{~mW}$ and $12 \mathrm{~V}$ respectively.

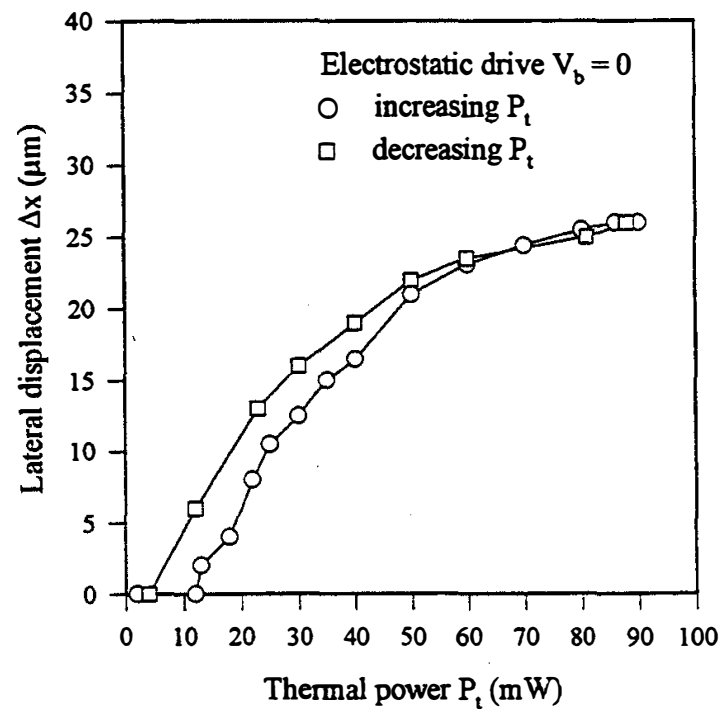

Fig.7. Measured lateral displacement as a function of input power for thermal actuation without electrostatic excitation.

Dynamic behavior of the actuator has been tested with a square-wave signal $\mathrm{V}_{\mathrm{a}}$ for the combined actuation. The results show that the actuation response depends largely on the tip motion amplitude. An up-bowed cantilever with an in-plane displacement amplitude of $15 \mu \mathrm{m}$ can respond at $360 \mathrm{~Hz}$. The cantilever with the full range in-plane amplitude of $30 \mu \mathrm{m}$ responds at approximate $10 \mathrm{~Hz}$. Our limited amount of fatigue testing shows that an up-bowed actuator with a vibration

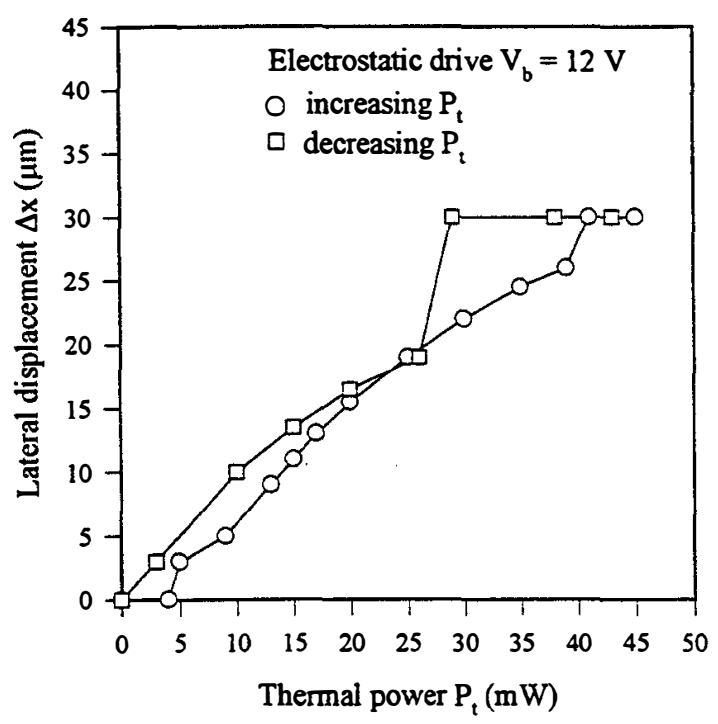

Fig.8. Measured lateral displacement as a function of input thermal power with combined actuation $\left(V_{a}\right.$ varies, $\left.V_{b}=12 \mathrm{~V}\right)$.

amplitude about $15 \mu \mathrm{m}$ will operate as long as several days continuously without obvious performance degradation; 
Actuators with the full range motion have operated only for tens of hours before failure.

Note that all the up-bowed actuators work well under the three drive conditions. The maximum lateral displacement achieved is up to $60 \mu \mathrm{m}$, determined by the initial shronk lateral displacement.

The adhesion between the movable tip part and the insulation layer is strongly affected by room ambient condition. Generally, after a several weeks storage in damp room air, the tip part is stuck to the substrate. In these cases tip performance can be recovered by repeating the release process again.

\section{CONCLUSIONS}

An up-bowed microactuator which achieved large lateral in-plane displacement up to $60 \mu \mathrm{m}$, was successfully designed and fabricated with standard CMOS fabrication process. Electrostatic, thermal, and the combined actuations were evaluated. The combined drive reduced the power supply requirements to a much lower level. The combined actuation was powered directly with CMOS circuitry. Hysteresis was observed among all three actuations and the tip fiction was considered to be the important hysteresis factor. The dynamic response and the lifetime for continuous operation of the devices depended largely on the vibration amplitude. With half range vibration amplitude the devices were operated at $360 \mathrm{~Hz}$ for several days without failure.

\section{ACKNOWLEDGMENT}

We would like to give thanks to Dr. Robert Marcus and Yanwei Zhang of Microelectronics Research Center, NJT for helpful suggestions.

\section{REFERENCES}

1. W.-H. Chu and M. Mehregany, 'Microfabricated tweezers with a large gripping force and a large range of motion", Technical Digest of the 1994 Solid-State Sensor and Actuator Workshop, Hilton Head, SC, June 13-16 (1996), pp. 107-110.

2. L. Chen, Z. Zhang, J. Bao, D. C. Thomas, and N. C. Macdonald, "Selective chemical vapor deposition of tungsten for microdynamic structures", Proc. IEEE MEM S-89, Salt Lake City, Utah, Fub. 20-22 (1989), pp. 83-87.

3. G. Lin, C.-J. Kim, et al, 'Design, fabrication, and testing of a C-shape actuator", Digest of Transducers'95, Stockholm, Sweden, June 25-29(1995), pp. 416-419.

4. H. J. Quenzer, A. Maciossek, et al, "Surface micromachined metallic microactuator with buckling characteristics", Digest of Transducers'95, Stockholm, Sweden, June 25-29 (1995), pp. 128-131.

5. C. G. Keller and R. T. Howe, "Nickel-filled hexsil thermally actuated tweezers", Digest of Transducers'95, Stockholm, Sweden, June 25-29 (1995), pp. 376-379.

6. X.-Q. Sun, Z.-J. Li, and L.-T. Liu, "Some structure improvements in silicon based micro electrostatic motors", Digest of Transducers'93, Yokohama, Japan, June 7-10 (1993), pp. 760-762.

7. G. Zheng and W. N. Carr, "Internal stress of thin bimorph microengineered structures", Proc. ICMPC'94, Kunming, China, Nov. 7-11 (1994), pp.498-503.
8. H. Jiang and W. N. Carr, "On-chip integration of high-voltage generator circuits for an electrostatic micromotor", Digest of Transducers'95, Stockholm, Sweden, June $25-29$ (1995), pp. 150-153,.

9. X.-Q. Sun and Z.-J. Li, "A micro polysilicon beam switch vibrator", Chinese Joumal of Semiconductors, Vol. 14, No. 6, pp. 320-327, 1993.

10. T. Aliyama and K. Shono, "Controlled stepwise motion in polysilicon microstructures", Joumal of Micro Electro Mechanical Systems, Vol. 2, No. 3, pp. 106-110, 1993. 